\title{
Characterizing Nonconstant Instrumental Variance in Emerging Miniaturized Analytical Techniques
}

Scott D. Noblitt, ${ }^{1 *}$, Kathleen E. Berg ${ }^{1}$, David M. Cate ${ }^{2}$, and Charles S. Henry ${ }^{1}{ }^{*}$

${ }^{1}$ Department of Chemistry, Colorado State University, Fort Collins, CO 80523

${ }^{2}$ School of Biomedical Engineering, Colorado State University, Fort Collins, CO 80523

*Corresponding authors

Scott.Noblitt@colostate.edu

Chuck.Henry@colostate.edu

Keywords:

calibration, weighted regression, precision, paper-based analytical device, cathodic stripping voltammetry, microchip electrophoresis 


\section{Abstract}

Measurement variance is a crucial aspect of quantitative chemical analysis. Variance directly affects important analytical figures of merit, including detection limit, quantitation limit, and confidence intervals. Most reported analyses for emerging analytical techniques implicitly assume constant variance (homoskedasticity) by using unweighted regression calibrations. Despite the assumption of constant variance, it is known that most instruments exhibit heteroskedasticity, where variance changes with signal intensity. Ignoring nonconstant variance results in suboptimal calibrations, invalid uncertainty estimates, and incorrect detection limits. Three techniques where homoskedasticity is often assumed were covered in this work to evaluate if heteroskedasticity had a significant quantitative impact-naked-eye, distance-based detection using paper-based analytical devices (PADs), cathodic stripping voltammetry (CSV) with disposable carbon-ink electrode devices, and microchip electrophoresis (MCE) with conductivity detection. Despite these techniques representing a wide range of chemistries and precision, heteroskedastic behavior was confirmed for each. The general variance forms were analyzed, and recommendations for accounting for nonconstant variance discussed. Monte Carlo simulations of instrument responses were performed to quantify the benefits of weighted regression, and the sensitivity to uncertainty in the variance function was tested. Results show that heteroskedasticity should be considered during development of new techniques; even moderate uncertainty (30\%) in the variance function still results in weighted regression outperforming unweighted regressions. We recommend utilizing the power model of variance because it is easy to apply, requires little additional experimentation, and produces higher-precision results and more reliable uncertainty estimates than assuming homoskedasticity. 


\section{Introduction}

Signal variance is intrinsic to all quantitative measurements in analytical chemistry. The variance, which is the square of the population standard deviation $(\sigma)$, determines both the limit of detection and limit of quantitation, which are often respectively defined as the concentrations yielding signals $3 \times$ and $10 \times$ the standard deviation of blank analyses. Similarly, variance governs the confidence intervals of individual measurements regardless if the confidence interval is propagated from calibration-curve results or estimated from replicate analyses. Consequently, measurement variance has a dominant role in both experimental design and reporting of results. Despite its importance, thorough characterization of measurement variance is frequently not performed or reported in the development of emerging analytical techniques. Literature publications often convey individual results as a value and sample standard deviation $(s)$ for a small $(\leq 6)$ number of replicate analyses $(n)$. The reported value is typically computed using a calibration curve calculated through ordinary least-squares regression (OLS). There are two problems with this approach; first, $s$ is a poor estimator for $\sigma$ at small sample sizes, which is partly due to the uncertainty of $\sigma$ following the $\chi^{2}$ distribution [1]. For example, the 95\% confidence interval for $s$ with $n=3$ is $-48 \%$ to $+528 \%$ of the nominal value; even for 12 replicates it is $-29 \%$ to $+70 \%$, which is a range that is equal in size to the best-estimate value itself (calculated using eq 12 , shown later). Consequently, the Student's t-test 95\% confidence intervals computed using $s$ from sample replicates are wider than for a single measurement when $\sigma$ is known until $n=4$ and are wider than $s$ itself until $n=7$. The second problem with the common reporting approach is that values are computed using OLS. OLS requires several assumptions for its output parameters to be valid, including 1) the true data trend is linear, 2) independent-variable errors are negligible, 3) measurements are independent of each other and their errors uncorrelated, 4) errors are normally distributed with a mean of zero, and 5) homoskedasticity. Although absolute validity of all five assumptions in real-world examples may never exist, proper experimental design and calibration can achieve close approximation of the first three assumptions, and the fourth assumption is also typically valid if no systematic errors are present. Homoskedasticity is therefore frequently the most common invalid assumption in 
analytical-chemistry applications. For instance, general instrument precision is often reported as the relative standard deviation (RSD) with the implication that it is approximately constant for a range of values. Indeed, RSDs frequently show only weak signal dependence well above the detection limit but can increase rapidly near the detection limit [2]. While OLS still provides unbiased estimates when presented with heteroskedastic data, it has suboptimal efficiency and the computed uncertainty values are invalid, particularly for $\mathrm{x}$-values far away from the calibration center point. This homoskedastic assumption of OLS is thus the focus of this work.

Homoskedastic regressions are improper for systems exhibiting near-constant RSDs since their uncertainties are signal dependent. Instead, weighted least-squares (WLS) regressions should be utilized, and the regression results can be used to establish uncertainty estimates from the calibration residuals. This method is more reliable than calculating confidence intervals based on estimates of $s$ from single-sample replicates because of the larger overall sample size. Thus, both common issues described above can be remedied through application of WLS. The primary difficulty in applying WLS to analytical data is lack of knowledge of the proper weightings (w). Minimum-variance estimation of the regression parameters occurs when $w$ is proportional to $\sigma^{-2}$. Unfortunately, the true variance is rarely known. Because of the aforementioned large uncertainty in $s$, setting $w$ proportional to $s^{-2}$ results in WLS underperforming OLS for few replicates $(n \leq 6)$ [3]. A better approach, called variance function estimation (VFE), fits the signal variance as a function of analyte concentration, and the resulting function is called the variance function [4, 5]. A variety of variance-function forms can be used [2, 4-8], and the choice depends on response characteristics and concentration range. Variance fitting can be performed on either regression residuals or from $s$ values calculated from replicate analyses. The residuals approach is theoretically superior, but the replicate method has been shown to be nearly as efficient and is not iterative [6]. Monte Carlo simulations have shown that acceptable estimates of the variance function can be achieved with as few as 24 data points (four replicates of six samples) [6]. Once the variance structure is known, the proper weighting form can be applied a priori in future calibrations, permitting proper WLS to be used with no more data collection than is necessary for OLS. 
Because methods for accommodating heteroskedasticity are established, the major hurdles to its inclusion in emerging analytical techniques are incomplete knowledge of the analytical system and unawareness of OLS limitations. This work partly addresses these roadblocks by characterizing the variance of three emerging miniaturized analytical techniques having dramatically different chemistries and precision levels, each of which has had little prior variance characterization by the research community—distance-based measurements of Ni using paper-based analytical devices (PADs) [9, 10], cathodic stripping voltammetry (CSV) of Mn using miniaturized electrochemical devices [11], and microchip electrophoresis (MCE) separation of anions using conductivity detection with internalstandard correction $[12,13]$. To the authors' knowledge, this is the first thorough characterization of heteroskedasticity in PADs, although previous work in our group utilized WLS for calibration [10]; that data set is revisited here. The authors are also unaware of any prior publications on the heteroskedasticity of carbon-ink CSV data; however, nonconstant variance in traditional stripping voltammetry instrumentation has been reported $[14,15]$. Heteroskedasticity in electrophoresis has more precedence. Early work discussing the heteroskedasticity of capillary electrophoresis was performed by Baumann and Wätzig using traditional capillary electrophoresis with absorbance detection [16]. However, only a handful of reports using electrophoresis utilize weighted regression [17-21], although some uses of WLS may have eluded literature searches. None of these electrophoresis reports used the microchip format, conductivity detection, or an internal standard. Previous work in our group employed weighted regression for analyses with similar MCE devices [13], but the variance was not thoroughly characterized and the weighting function was only estimated.

Each technique tested in this work was observed to exhibit heteroskedasticity and was characterized using VFE. Empirical results were used for Monte Carlo simulations of the instruments’ performance to more fully characterize the importance of accounting for heteroskedasticity. Sensitivity to uncertainty in variance function was also assessed. Results indicated that heteroskedasticity should be considered in experimental designs for each technique. Benefits of weighted regression were particularly strong at the low end of calibration ranges, prediction bands were more accurate with WLS, and moderate 
uncertainty $( \pm 30 \%)$ in the variance function only led to a small drop in performance, with WLS still outperforming OLS.

\section{Theory}

Both OLS and WLS are well-established techniques described in analytical-chemistry literature [7, 2224]. Relevant equations will be covered in a format familiar to most practicing analytical chemists. The calibration function of interest for both OLS and WLS is the basic linear function eq 1, where $y$ is the dependent variable (signal), $x$ is the independent variable (e.g. concentration or mass), $m$ is the slope (sensitivity), and $b$ is the intercept (signal for the blank or negative control).

$$
y=m x+b
$$

For a set of $N$ total observations (not to be confused with individual-sample replicates, $n$ ) of $y$ for various $x, m$ and $b$ can be estimated using least-squares regression. These are calculated with eqs $2-3$.

$$
\begin{aligned}
& m=\frac{S_{x y}}{S_{x x}} \\
& b=\bar{y}-m \bar{x}
\end{aligned}
$$

The weighted $S_{x x}$ and $S_{x y}$ are calculated using eqs 4-5, and the weighted $x$ - and y-centroids (the values with overlines) are given in eqs 6-7. The subscript $i$ represents an independent calibration observation. For each equation, weights $(w)$ are scaled so their sum equals $N$, and the OLS equivalent is achieved by replacing $w$ with a unity value.

$$
\begin{aligned}
& S_{x x}=\sum_{i=1}^{N} w_{i}\left(x_{i}-\bar{x}\right)^{2} \\
& S_{x y}=\sum_{i=1}^{N} w_{i}\left(x_{i}-\bar{x}\right)\left(y_{i}-\bar{y}\right)
\end{aligned}
$$




$$
\begin{gathered}
\bar{x}=\frac{\sum_{i=1}^{N} w_{i} x_{i}}{\sum_{i=1}^{N} w_{i}} \\
\bar{y}=\frac{\sum_{i=1}^{N} w_{i} y_{i}}{\sum_{i=1}^{N} w_{i}}
\end{gathered}
$$

After fitting a line to the data, $\mathrm{SS}_{\mathrm{E}}$ (sum of the squared residuals) can be calculated using eq 8 with the expected signals from the calibration $(\hat{y})$. Eq 9 is then used to calculate the weighted residual standard deviation, $\hat{s}$, where DOF stands for degrees of freedom (normally $N-2$ ).

$$
\begin{aligned}
& S S_{E}=\sum_{i=1}^{N} w_{i}\left(y_{i}-\hat{y}\right)^{2} \\
& \hat{s}=\sqrt{\frac{S S_{E}}{D O F}}
\end{aligned}
$$

The confidence bands and prediction bands (PB) around the best-fit line are computed using eqs 10 and 11, respectively, where the subscript $o$ indicates the position of interest (not necessarily a calibration point) and $t_{\alpha / 2, D O F}$ is the upper $\alpha / 2$ fraction of the $t$ distribution for the DOF.

$$
\begin{aligned}
& C B= \pm t_{\frac{\alpha}{2}, D O F} \hat{s} \sqrt{\frac{1}{N}+\frac{\left(x_{o}-\bar{x}\right)^{2}}{S_{x x}}} \\
& P B= \pm t_{\frac{\alpha}{2}, D O F} \hat{s} \sqrt{\frac{1}{w_{o}}+\frac{1}{N}+\frac{\left(x_{o}-\bar{x}\right)^{2}}{S_{x x}}}
\end{aligned}
$$

The uncertainty provided in eq 11 is for a single measurement and is more reliable than using $s$ calculated from individual measurement replicates (assuming proper regression weighting and $N>n$ ). 
While eqs 1-11 are straightforward (and therefore included in commercial software packages), acquiring appropriate values for $w$ requires fitting the variance function to calibration data, which is less clear-cut. The major difficulty in evaluating the variance function is that $s$ estimates from replicates follow the $\chi^{2}$ distribution, meaning large and highly asymmetrical uncertainties for low $n$. The confidence interval around $\sigma$ calculated from $s$ is given by eq 12 , where $\chi_{\alpha / 2, \mathrm{~N}-1}^{2}$ and $\chi_{1-\alpha / 2, \mathrm{~N}-1}^{2}$ are the upper and lower fractions of the $\chi^{2}$ distribution, respectively, for a confidence level of $1-\alpha$.

$$
\sqrt{\frac{(N-1) s^{2}}{\chi_{\alpha / 2, N-1}^{2}}} \leq \sigma \leq \sqrt{\frac{(N-1) s^{2}}{\chi_{1-\alpha / 2, N-1}^{2}}}
$$

This uncertainty for the standard deviation has the convenient benefit of being proportional to the estimated standard deviation itself, making appropriate weighting of the variance function easy [1]. Previous work by Tellinghuisen showed properly weighting the variance-function fit still yielded an unbiased, minimum-variance estimate of the parameters despite the non-Gaussian distributions [1]. A wide range of variance functions have been proposed historically; in this work, four common variance functions were considered, shown in eqs 13-16, where $c_{0}$ and $c_{1}$ are adjustable constants.

$$
\begin{aligned}
& \sigma_{y}=c_{0}+c_{1} y \\
& \sigma_{y}^{2}=c_{0}+c_{1} y^{2} \\
& \sigma_{y}^{2}=c_{0}+c_{1} y \\
& \sigma_{y}=c_{0} y^{c_{1}}
\end{aligned}
$$

Appropriate weightings for WLS are proportional to $\sigma^{-2}$. Each equation has its own merits and deficiencies. Although eq 13 has been proposed in several works, it conflicts with theoretical expectations of variances, not standard deviations, being additive. Eqs 14-15 model the variance as a sum of sources and can be described as components of variance models [5]. Eqs 13-15 have fixed, integer exponents for their relationship to $\sigma$ or $\sigma^{2}$, but there is no theoretical requirement for an integer 
exponent, and previous work has shown that fractional exponents can describe real systems [25]. Eq 16 is termed the power model and is easy to employ, yielding results that effectively only depend upon $c_{1}$ because $c_{0}$ affects all weights equally. However, eq 16 conflicts with the accepted definition of limit of detection ( $3 \sigma$ of the blanks) because it implies zero variance at zero signal. Examples of eqs 13-16 are plotted in supporting information Figure S1 showing the general shapes of the functions. Finally, eqs 13-16 all model $\sigma$ as a function of $y$, but analogous models can also use $x$.

\section{Experimental}

Materials

A full list of chemicals used in this work and their sources is given in the supporting information. All chemicals were of reagent grade or higher and used as provide; solutions were prepared using 18.2 $\mathrm{M} \Omega \cdot \mathrm{cm}$ water generated from a Milli-Q (Merck Millipore, Darmstadt, Germany) purifier.

\section{Instrumentation}

Because each technique used in this work has been previously published, detailed experimental information is not covered. Instead, brief descriptions are provided in the supporting information and short summaries are given here. The PAD devices employed colorimetric detection for Ni using travel distance as the quantitative signal [10]. Wax barriers to constrain fluid flow were printed (Xerox ColorQube 8870, Norwalk, CT, USA) in a thermometer-like shape onto Grade 1 Whatman filter paper (Apollo Presentation Products, Booneville, MS, USA). Analyte signal distance (length of colored complex) was quantified using a ruler printed on the substrate. Representative results for these devices and chemistry are shown in Figure 1a. The CSV carbon-ink device was used for Mn analysis [11]. Carbon ink (E3178, Ercon Inc., Wareham, MA, USA) electrodes were printed onto transparency film (3M, St. Paul, MN, USA). Measurements were taken in $60 \mu \mathrm{L}$ of a $50 \mathrm{mM}$ pH 5.0 acetate buffer 
containing 3.5\% NaCl. Electrochemistry was performed using an eDAQ (Denistone East, Australia) EA161 Potentiostat and EC201 e-Corder. Square-wave stripping voltammetry parameters used a deposition potential and time of $+0.85 \mathrm{~V}$ and $180 \mathrm{~s}$, incremental potential of $4 \mathrm{mV}$, amplitude of 105 $\mathrm{mV}$, frequency of $15 \mathrm{~Hz}$, and final potential of $-1 \mathrm{~V}$. The calibration quantity was the Mn peak height. Example calibration voltammagrams and the electrode layout are shown in Figure 1b. For the MCE analysis, devices were prepared using a Sylgard 184 elastomer/crosslinker kit (Dow Corning, Midland, MI, USA) and standard soft lithography techniques [26]. Contact conductivity detection was performed using a bubble-cell detection zone [27], specific microfluidic geometry was used previously [28], and injection mode was gated injection [29]. The MCE separation electrolyte was composed of $40 \mathrm{mM}$ picolinic acid, $26 \mathrm{mM}$ isonicotinamide, $4.6 \mathrm{mM}$ N-(2-hydroxyethyl)piperazine-N'-(4-butanesulfonic acid), and $18 \mathrm{mM}$ N-tetradecyl-N,N-diemthyl-3-ammonio-1-propanesulfonate, which was derived as a combination of two previously reported separation electrolytes [12, 13]. Analyte order was chloride, sulfate, selenate, nitrate, 1,2-ethanedisulfonate (internal standard), and oxalate. The quantity of interest was the analyte/internal standard peak area ratio. Representative separations and the microfluidic layout are shown in Figure 1c. 


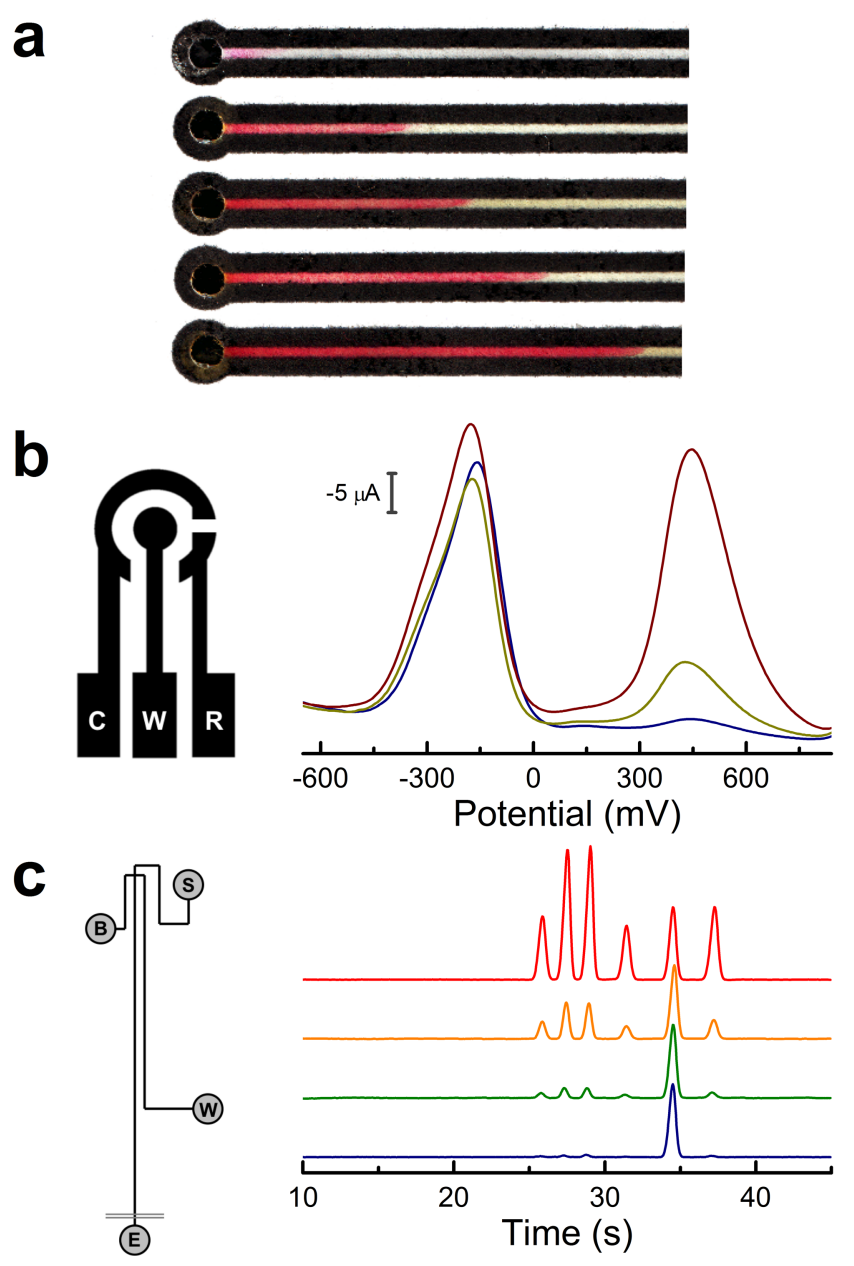

Figure 1. Device depiction and representative data for (a) distance-based Ni PAD, (b) disposable carbon-ink CSV for Mn, and (c) MCE anion analysis. Ni PADs are shown as photographs of devices sampling concentrations ( $\mu \mathrm{g}$, top to bottom) of 0.10, 2.3, 7.0, 8.5 and 12. CSV depiction shows the working electrode (W), counter electrode (C), and reference electrode (R). Mn CSVs show benzoquinone (standard species) at $-200 \mathrm{mV}$ and $\mathrm{Mn}$ at $+450 \mathrm{mV}$ for $\mathrm{Mn}$ concentrations ( $\mu \mathrm{M}$, bottom to top) of 2.0, 9.0, and 25. MCE device shows the following reservoirs: sample (S), waste (W), buffer (B), and separation (E). Separation order was chloride, sulfate, selenate, nitrate, 1,2-ethanedisulfonate (internal standard), and oxalate. Internal standard concentration was $10 \mu \mathrm{M}$, and analyte concentration ( $\mu \mathrm{M}$, bottom to top) was $0.25,1.0,3.7$, and 15 .

\section{Data Processing}

In this work, replicate analysis was used to estimate the variance function. For methods fitting $\sigma^{2}$ (eqs 14-15), empirical $s^{2}$ values were weighted by $s^{-4}$ in linear regressions. For eq 13 fitting $\sigma$ using $s$ values, weightings were $s^{-2}$. Weightings were suggested by Tellinghuisen and are based on the proportionality of the uncertainty in $s$ with the same number of replicates (eq 12) [1]. For eq 16, a logarithmic transformation was performed, followed by OLS to estimate parameters. The logarithmic transformation 
is known to lead to a small bias [1], but the bias is inconsequential for many applications. Regressions were performed using a custom LabVIEW program that was verified with several data sets using Microsoft Excel. Plots were generated using OriginPro 8.0 software.

\section{Monte Carlo Simulations}

LabVIEW was used to perform simulations of instrumental runs. Slope, intercept, and independent variables were input for six-point calibrations. Noise was added to each calibration point according to the user-input variance function (based on eq 16 VFE fits to empirical results) using the Gaussian White Noise LabVIEW virtual instrument, which generates pseudo-random numbers. The resulting six-point calibration sets were analyzed by OLS, WLS with the proper $c_{1}$ value from eq 16 , and WLS using $\pm 30 \%$ of the correct $c_{1}$. Slopes and intercepts were recorded for each regression. Two user-input sample points with appropriate noise from Gaussian White Noise were evaluated with each regression method,

yielding a best estimate and 95\% prediction interval uncertainty. Each simulation was performed $10^{5}$ times.

\section{Results and Discussion}

\section{Distance-Based PAD}

Variance as a function of response was evaluated for the distance-based PAD analysis of Ni by constructing a calibration curve using Ni masses of 5.0, 15, 25, 35, 45, and $55 \mu \mathrm{g}(n=32, N=192)$. The expected RSD values are $\approx 7 \%$ [10]. Contributors to uncertainty include device manufacturing reproducibility (each device is used once and discarded), pipetting precision ( $\approx 1 \% \mathrm{RSD}$ ), and the unaided-eye reading precision, which is truncated to the nearest $0.5 \mathrm{~mm}$ and therefore not a continuous distribution. Another important aspect of this technique is its positive y-intercept value, which has 
implications at the low end of the calibration curve when weighting based upon the measured signal (instead of concentration or mass).

Results from the VFE and regression analyses are shown in Table 1. A plot of the variance fits is shown in supporting Figures S2-S4. Differences in the measured slopes and intercepts between the methods are minor, which is a product of the large $N$ and each method providing ideally unbiased estimates. While the negative $c_{0}$ value for eq 15 is concerning, in practice a negative (impossible) variance is never realized because the calibration intercept is positive enough that the variance always remain positive. Also of note is the $c_{1}$ value for eq 16 , which indicates that standard deviation goes up nearly linearly with concentration and therefore yields a nearly constant RSD.

Table 1. Fitting parameters obtained from VFE and linear regression.

\begin{tabular}{|l|l|l|l|}
\hline VFE Eq & Ni(II) PAD & Mn(II) CSV & Se(VI) MCE \\
\hline 13 & $\mathrm{c}_{0}=0.062$ & $\mathrm{c}_{0}=0.28$ & $\mathrm{c}_{0}=0.0022$ \\
& $\mathrm{c}_{1}=0.071$ & $\mathrm{c}_{1}=0.056$ & $\mathrm{c}_{1}=0.0032$ \\
& $\mathrm{~m}=0.505$ & $\mathrm{~m}=1.34$ & $\mathrm{~m}=1.128$ \\
& $\mathrm{~b}=3.72$ & $\mathrm{~b}=-1.46$ & $\mathrm{~b}=0.0003$ \\
\hline 14 & $\mathrm{c}_{0}=0.026$ & $\mathrm{c}_{0}=0.098$ & $\mathrm{c}_{0}=5.4 \times 10^{-5}$ \\
& $\mathrm{c}_{1}=0.0056$ & $\mathrm{c}_{1}=0.0037$ & $\mathrm{c}_{1}=1.8 \times 10^{-5}$ \\
& $\mathrm{~m}=0.505$ & $\mathrm{~m}=1.33$ & $\mathrm{~m}=1.128$ \\
& $\mathrm{~b}=3.72$ & $\mathrm{~b}=-1.42$ & $\mathrm{~b}=0.0003$ \\
\hline 15 & $\mathrm{c}_{0}=-0.87$ & $\mathrm{c}_{0}=-0.042$ & $\mathrm{c}_{0}=4.0 \times 10^{-6}$ \\
& $\mathrm{c}_{1}=0.17$ & $\mathrm{c}_{1}=0.092$ & $\mathrm{c}_{1}=2.3 \times 10^{-5}$ \\
& $\mathrm{~m}=0.514$ & $\mathrm{~m}=1.36$ & $\mathrm{~m}=1.129$ \\
& $\mathrm{~b}=3.54$ & $\mathrm{~b}=-1.51$ & $\mathrm{~b}=0.0002$ \\
\hline 16 & $\mathrm{c}_{0}=0.098$ & $\mathrm{c}_{0}=0.30$ & $\mathrm{c}_{0}=0.0063$ \\
& $\mathrm{c}_{1}=0.91$ & $\mathrm{c}_{1}=0.55$ & $\mathrm{c}_{1}=0.31$ \\
& $\mathrm{~m}=0.506$ & $\mathrm{~m}=1.36$ & $\mathrm{~m}=1.129$ \\
& $\mathrm{~b}=3.71$ & $\mathrm{~b}=-1.51$ & $\mathrm{~b}=0.0000$ \\
\hline OLS & $\mathrm{m}=0.519$ & $\mathrm{~m}=1.41$ & $\mathrm{~m}=1.129$ \\
& $\mathrm{~b}=3.57$ & $\mathrm{~b}=-1.88$ & $\mathrm{~b}=-0.0003$ \\
\hline
\end{tabular}

While the computed slopes and intercepts show little difference between OLS and individual VFE methods, calculated uncertainties (as PBs) strongly differ with WLS. A plot of the residuals (from OLS) and PBs for OLS and each WLS is shown in Figure 2a. The importance of including heteroskedasticity 
in the regression is clear, with OLS uncertainty being strongly overestimated at the low end of the calibration and underestimated at the high end. Quantitatively, $s$ for the lowest concentration was 0.48 $\mathrm{mm}$ (close to the 0.5-mm measurement resolution), but the 95\% OLS prediction interval was $\pm 3.4 \mathrm{~mm}$, or $7.0 \mathrm{~s}$ instead of the expected $\approx 2 \mathrm{~s}$. At the highest concentration, observed $s$ was $2.5 \mathrm{~mm}$, whereas the OLS prediction interval was again $\pm 3.4 \mathrm{~mm}$. This prediction interval was $1.3 \mathrm{~s}$ instead of $\approx 2 s$ and therefore equivalent to a roughly $82 \%$ prediction interval. Thus, the underestimation of uncertainty at the high range was significant but less erroneous than the extreme overestimation at the low range. In contrast, each of the VFE equations yielded prediction bands that followed the uncertainty trend well despite significant differences in the variance-function forms. The only major difference between methods comes from eq 15, which shows a marked decrease in the prediction interval at the bottom of the calibration range due to its negative $c_{0}$ value. The agreement between functions is likely due to the small range of concentrations (a factor of 11) used in the analysis, but this is nearly the full linear range of the method. The average prediction interval using the four equations for the lowest-concentration point was $\pm 1.0 \mathrm{~mm}(2.2 \mathrm{~s})$. The average highest-concentration prediction interval was $\pm 4.9 \mathrm{~mm}(1.9 \mathrm{~s})$. Consequently, it was concluded that any of the VFE functions would be adequate for this technique, and each would be superior to OLS. If further significant improvements are desired, then the measurement's discrete nature would likely need to be included in the statistical analysis. 

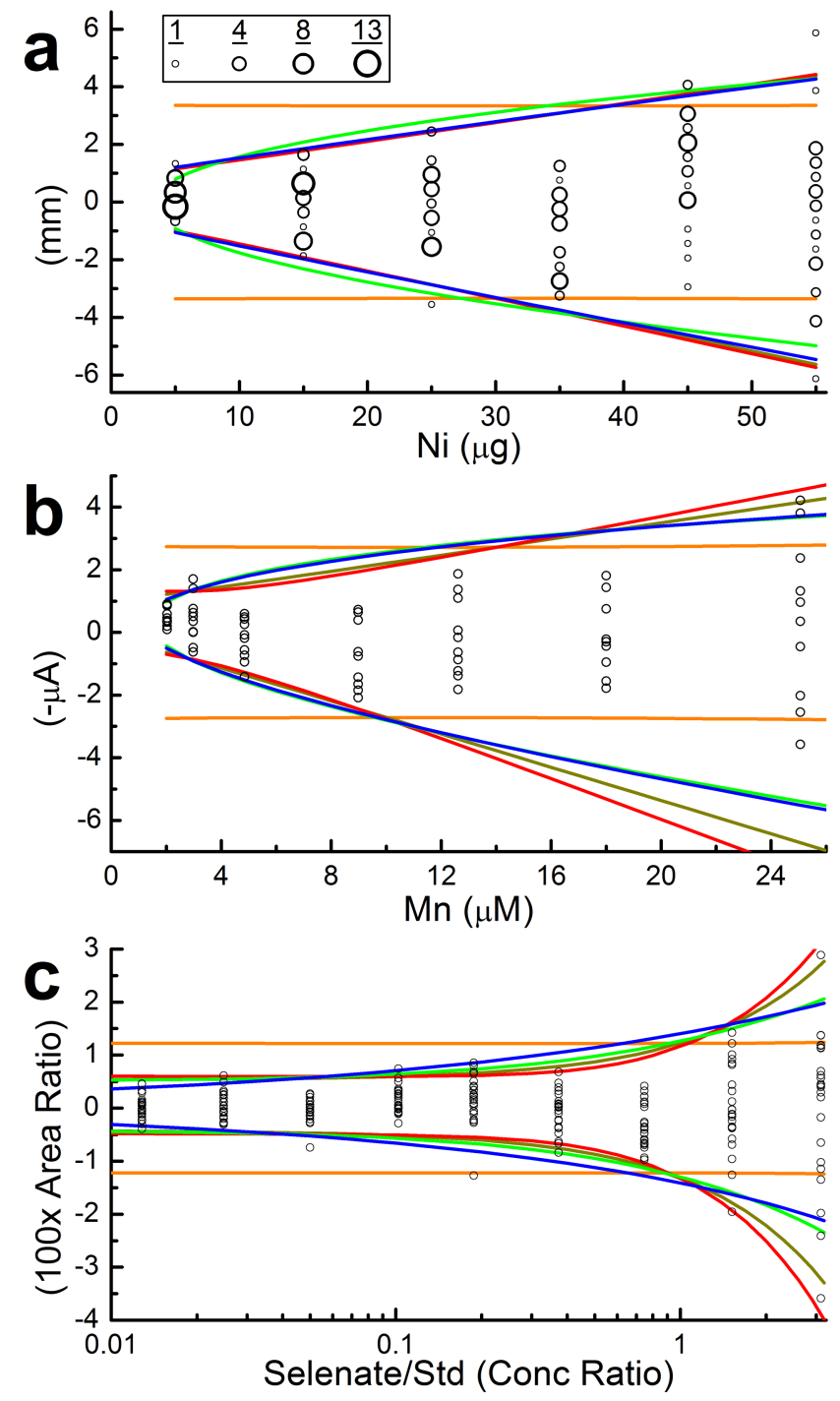

Figure 2. Calibration residuals including 95\% prediction intervals for OLS (orange) and WLS fits using VFE with eq 13 (dark yellow), eq 14 (red), eq 15 (green), and eq 16 (blue). a) Ni PAD results; because the data are discretely distributed at $0.5-\mathrm{mm}$ increments, plotted point sizes are scaled proportionally to the number of observations at each value to better reflect the data distribution. b) Mn CSV results. c) Selenate MCE results using $\log _{10}$ scale.

To quantitatively assess the superiority of WLS and establish its sensitivity to VFE uncertainty, Monte Carlo simulations were performed using the Ni PAD results. The assumed slope and intercept were 0.51 $\mathrm{mm} \mu \mathrm{g}^{-1}$ and $3.7 \mathrm{~mm}$, respectively, which are similar to measured values using both OLS and WLS. Variance was modeled using eq 16 with $c_{0}=0.0096^{0.5}$ and $c_{1}=0.90$. Calibration concentrations were the same as used experimentally, and test concentrations were $10 \mu \mathrm{g}$ and $50 \mu \mathrm{g}$ (representing the low and high end). Regression analyses were performed with OLS and WLS assuming weights of $\mathrm{y}^{-1.8}$ (correct), $\mathrm{y}^{-1.26}$ (30\% low), and $\mathrm{y}^{-2.34}$ (30\% high). Histograms for the OLS and correct WLS prediction 
intervals are shown in Figure 3a-b. Complete graphical results for all weightings tested for the slope, intercept, best-estimate values, and prediction intervals are shown in supporting information Figures S9S10. Simulations confirmed the better efficiency of WLS; compiled simulation slopes had a $21.0 \%$ lower standard deviation than OLS values (0.0293 vs. 0.0371). The lower standard deviation with WLS manifests as higher-precision values for experimental calibrations. Improvements in intercept precision were even better, with a $32.8 \%$ decrease in standard deviation (0.561 vs. 0.835$)$. The greater improvement in intercept measurement precision is due to the increased weight given to the higher precision low-concentration points. Uncertainty in the weights did surprisingly little to reduce the improved efficiency of WLS-the $\mathrm{y}^{-1.26}$ and $\mathrm{y}^{-2.34}$ weightings gave $19.7 \%$ and $18.6 \%$ lower standard deviations for slopes than OLS and were 30.9\% and 31.9\% lower for intercepts, respectively. The lack of a large performance drop with incorrect weighting has been observed previously [3], and represents a convincing argument for using WLS despite uncertain variance functions. Interestingly, each of the weighted results yielded a slightly negative bias for the measured slope, averaging $0.9 \%$ low. However, this systematic bias has little practical relevance because the experimental method has a near-constant RSD of about $7 \%$. 

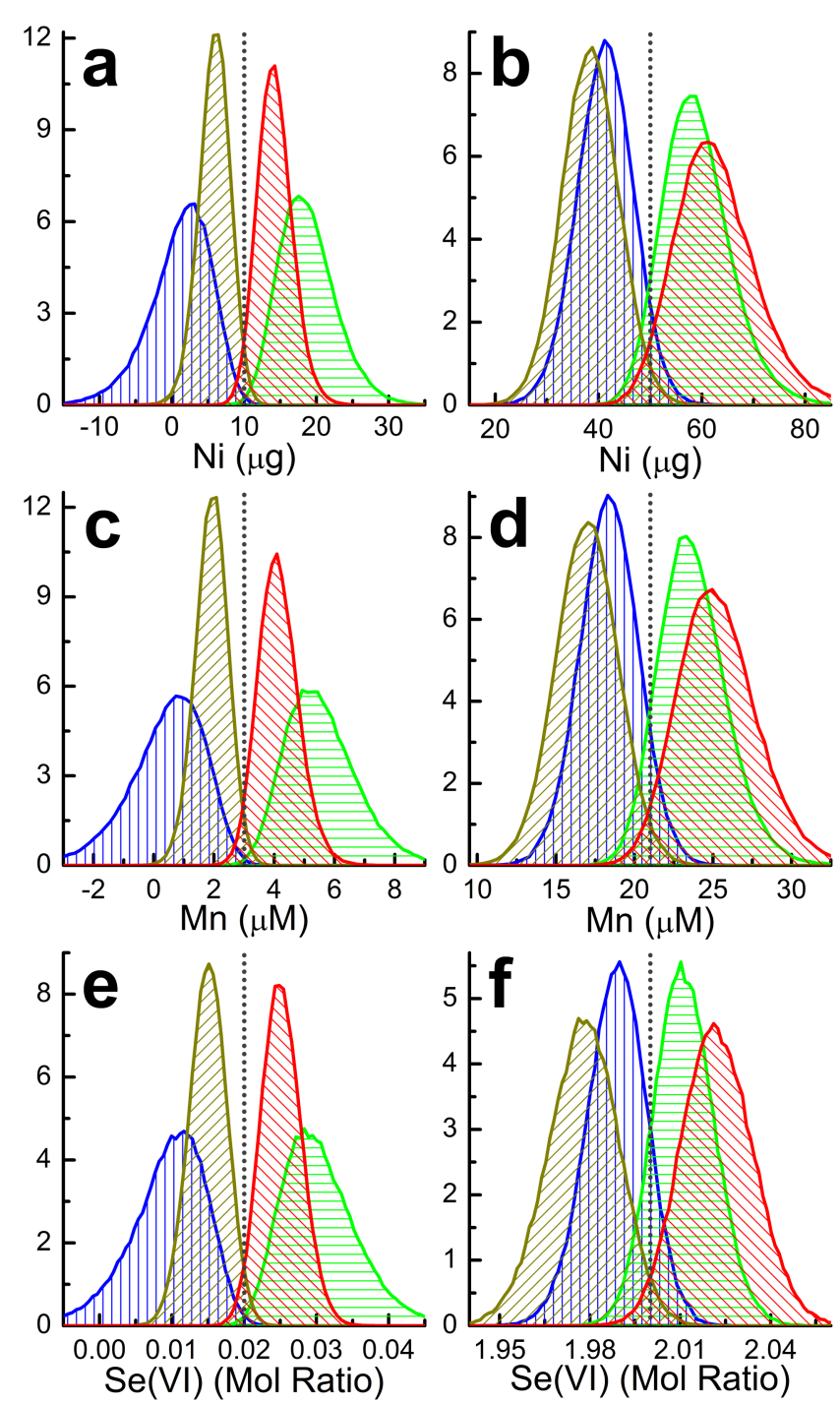

Figure 3. Histogram representations of the $10^{5}$ Monte Carlo simulations for the representative low (left) and high (right) concentrations. The y-axes are histogram counts. OLS lower prediction limit = blue, OLS upper prediction limit = green, WLS lower prediction limit = dark yellow, WLS upper prediction limit $=$ red, and actual value $=$ dotted gray line. $\mathrm{a}-\mathrm{b}) \mathrm{Ni}$ PAD results; histograms used 150 bins with a bin width of $0.653 \mu \mathrm{g}$ for the low concentration and $1.18 \mu \mathrm{g}$ for the high concentration. c-d) Mn CSV results; histograms used 150 bins with bin widths of $0.168 \mu \mathrm{M}$ for the low concentration and $0.416 \mu \mathrm{M}$ for the high concentration. e-f) Selenate MCE results; histograms used 150 bins with bin widths of $5.73 \times 10^{-4}$ for the low ratio and $1.36 \times 10^{-3}$ for the high ratio.

Low-concentration simulation results confirmed that uncertainty was too conservative using OLS. Only $0.6 \%$ of the OLS simulations found the low-concentration point outside of the prediction interval versus 5.1\% using correct WLS (5.0\% is the expected value for 95\% confidence). Incorrect weighting led to qualitatively predictable shifts, with $\mathrm{y}^{-1.26}$ and $\mathrm{y}^{-2.34}$ weightings yielding $2.7 \%$ and $8.9 \%$ of the data points falling outside prediction intervals, respectively. The precision of WLS evaluations was also 
superior to OLS. WLS standard deviations for the low-concentration point were 9-10\% lower. Simulations of high concentrations showed WLS to be superior to OLS, but the differences were not as severe. As expected, OLS uncertainty was too liberal, with $12.5 \%$ of simulations falling outside the prediction intervals compared to $5.2 \%$ for correct WLS. Weightings of $\mathrm{y}^{-1.26}$ and $\mathrm{y}^{-2.34}$ yielded $6.8 \%$ and 4.0\%, respectively. Precision improvements were of no practical benefit at only $0.1-1.8 \%$ for standard deviation. Overall, it can be concluded that WLS improvements are more significant at the low end of concentration range than the high end, with the only major benefit at the high end being improved prediction intervals.

\section{Cathodic Stripping Voltammetry}

The variance function for Mn CSV using carbon-ink devices was evaluated using concentrations of 2.0, 3.0, 5.0, 9.0, 12.5, 18, and $25 \mu \mathrm{M}(n=10, N=70)$. Like the visual PAD with $\mathrm{Ni}$, RSDs were relatively high (>5\%) primarily because each device was only used once. Unlike the Ni PAD, measurement values should follow a continuous distribution because they stem from software peak-height measurements. Also in contrast to the Ni PAD, the calibration y-intercept is significantly negative.

Results from VFE and regression are shown in Table 1, and a plot of the variance-function fitting is shown in supporting Figures S11-S12. There was less agreement between OLS and WLS in this case than with the Ni PAD study, possibly due to the smaller $N$. The average WLS slope was $4.4 \%$ lower than OLS. The WLS intercepts were also significantly less negative than that from OLS. As with the Ni PAD, eq 15 was fit with $c_{0}$ being negative. Although alarming, estimated variance will not become negative in practice because $2 \mu \mathrm{M}$ is approximately the lower limit of linearity, so the variance function will not extend below this concentration. However, it is cause for skepticism towards the applicability of eq 15 for both the Ni PAD and Mn CSV systems. The $c_{1}$ value for eq 16 indicates that variance is approximately proportional to measured concentration, so RSD values will follow an approximately square-root relationship with concentration. Interestingly, the $c_{1}$ results from eq 16 would indicate that 
the relationship between variance and concentration might be modeled by eq 15 better than eqs 13-14, yet the negative $c_{0}$ value was still obtained for eq 15 .

The primary benefit of employing WLS is apparent by comparing prediction intervals. Figure 2b shows the OLS residuals for Mn CSV along with prediction intervals for all employed methods. Because estimated standard deviations from only 10 replicates have high uncertainty, the quantitative improvement in prediction-interval size was evaluated by averaging the two standard deviations and prediction-interval widths for the two lowest- and highest-concentration points. The average $s$ for 2-3 $\mu \mathrm{M}$ Mn was $0.52 \mu \mathrm{A}$. OLS and average WLS prediction intervals were $\pm 2.7 \mu \mathrm{A}(5.3 s)$ and $\pm 0.99 \mu \mathrm{A}$ (1.9s), respectively, where $\approx 2 s$ is expected. For the $18-25 \mu \mathrm{M} \mathrm{Mn}$, average $s$ was $1.9 \mu \mathrm{A}$, OLS prediction interval was $\pm 2.8 \mu \mathrm{A}(1.4 s)$, and WLS prediction interval was $\pm 4.3 \mu \mathrm{A}(2.3 s)$. As with the Ni PAD data, accounting for the heteroskedasticity improved the accuracy of the calculated uncertainties. Unlike for the Ni data, the choice of the VFE produced noticeably different PB shapes. Eqs 15 and 16 yielded almost identical results but are distinctly different from eq 14, while the bands from eq 13 appear between the ones from eq 14 and eqs 15/16. Determining which band shape is most appropriate is probably not possible with this data set. Ideally, extending the data to either higher or lower concentrations would be the best option for determining the proper VFE form; unfortunately, the calibration uses most of the linear range for this analysis, removing that option. Instead, a larger number of replicate analyses would be needed. An additional confounding problem is that the underlying behavior of the system may not be strictly linear as noted from residuals analysis leaving an approximately parabolic set of residuals with a minimum near $10 \mu \mathrm{M}$. Considering the short range of the data (factor of $12.5 \times$ ) and possible curvature, it may be most pragmatic for real-world calibrations to simply use eq 16 with a known $c_{1}$ determined previously from replicate analysis, as the result will still likely be representative of the data and only involves one relevant VFE fitting variable $\left(c_{1}\right)$. Another option would be to fit replicate data covering a larger range to a polynomial curve and perform VFE on the resulting residuals. 
Monte Carlo simulations of the Mn CSV system were performed to further evaluate the improvements yielded from WLS and the effect of VFE uncertainty. The assumed slope was $1.36-\mu \mathrm{A} / \mu \mathrm{M}$ with an intercept of $-1.51-\mu \mathrm{A}$. Variance was modeled using eq 16 with $c_{0}=0.089^{0.5}$ and $c_{1}=0.55$. Calibration concentrations $(\mu \mathrm{M})$ were $2,4,7,11,17$, and 25 ; test concentrations were $3 \mu \mathrm{M}$ and $21 \mu \mathrm{M}$. Regression analyses were performed with OLS and WLS assuming weights of $\mathrm{y}^{-1.1}$ (correct), $\mathrm{y}^{-0.77}$ (30\% low), and $\mathrm{y}^{-1.43}$ (30\% high). Histograms for OLS and correct WLS prediction intervals are shown in Figure 3c-d. Complete graphical results for the slope, intercept, best-estimate values, and prediction intervals using each regression approach are available in the supporting information. Results were qualitatively similar to those in the Ni PAD simulations. Calculated WLS slopes had a slight negative bias, on average $0.48 \%$, and the average WLS intercept was also biased low at -1.53 . These systematic biases are negligible compared to the method's random variance. Additionally, the small systematic bias is minor compared to the improvement in precision due to WLS. Optimal WLS yielded a $21.6 \%$ smaller standard deviation for the slope than OLS, and $\mathrm{y}^{-0.77}$ and $\mathrm{y}^{-1.43}$ weightings were $19.5 \%$ and $18.8 \%$ smaller, respectively. As with the Ni simulations, improvements in measured intercept versus OLS were even better, at $43.1 \%, 40.3 \%$, and $42.5 \%$ for $\mathrm{y}^{-1.1}, \mathrm{y}^{-0.77}$, and $\mathrm{y}^{-1.43}$ weightings, respectively. When analyzing the simulated measurement points, standard deviations for the low-concentration points were about $16 \%$ lower than OLS for each form of WLS. At the high end, precision was $<3 \%$ better. OLS found $0.5 \%$ of the low-concentration values to be outside of the prediction interval, whereas $16.9 \%$ of the highconcentration values were outside. WLS with weightings of $\mathrm{y}^{-1.1}, \mathrm{y}^{-0.77}$, and $\mathrm{y}^{-1.43}$ resulted in 5.1\%, 2.5\%, and $9.5 \%$ of the low-concentration measurements falling outside the prediction intervals, respectively. The respective high-end rates were 5.0\%, 7.6\%, and 3.4\%. Consistent with the Ni PAD analysis, uncertainties calculated from OLS are too conservative at low concentrations and too liberal at high concentrations. Proper WLS corrects for this, and even adding 30\% uncertainty to the weighting factor gave results that were superior to OLS. 
The variance function for the five inorganic anions in MCE with conductivity detection was evaluated using nine concentrations $(\mu \mathrm{M})$ of approximately $0.125,0.25,0.50,1.00,1.88,3.75,7.5,15$, and 30 Each solution was tested eight times, and each test consisted of three replicate injections for a total $N=$ 216. These measurements are not strictly independent because total liquid-handling events were only one third of the total measurements, but the assumption of independence is usually acceptable, particularly for non-ubiquitous ions (selenate and oxalate). Calibrations were based on peak area relative to the $10 \mu \mathrm{M}$ internal standard, 1,2-ethanedisulfonate. Inclusion of the internal standard corrects for several injection-related variances, greatly improving reproducibility [30, 31]. Additionally, the same MCE device is reusable for both calibration and sample analyses, removing the device-to-device contribution to variance, although utilization of the internal standard may eliminate most device-todevice variability [28, 32]. Through the use of an internal standard, capillary electrophoresis can achieve RSD values of $1 \%$ or better [33]. At these low levels of uncertainty, variance can easily be dominated by non-instrumental factors, such as the operator, standard- and sample-preparation procedures, fluctuations in ubiquitous-species contamination, matrix effects, peak integration protocols, and room-temperature variations. However, uncertainties of $\approx 1 \%$ can be valid under controlled conditions; for instance, selenate was properly measured with an uncertainty of $\approx 1 \%$ in a matrix originating from biological broth even though the calibration curve was prepared in only background electrolyte [13]. Consequently, the MCE system represents a significantly different technique than the PAD and CSV devices from a statistical perspective.

Results from VFE and regression are shown for selenate in Table 1. A plot of the selenate variance fits is shown in supporting Figures S13-S14. For brevity, results for chloride, sulfate, nitrate, and oxalate are provided in supporting information Figures S5-S8. Because of the high precision of the analysis (unweighted $\mathrm{R}^{2}=0.99997$ ), all of the slopes and intercepts for the various analysis approaches are practically identical. As with the PAD and CSV techniques, the primary benefit of employing WLS is through the improved PBs. A plot of the PBs overlaid on the OLS residuals is shown in Figure 2c, which is displayed in $\log _{10}$ scale for ease of reading two orders of magnitude. As with the other 
analyses, each WLS significantly outperforms OLS for generating PBs, but distinguishing between various WLS methods is more difficult. To help quantify the differences, average $s$ for the two lowest concentrations and two highest concentrations were compared to their average prediction-interval widths, as was done with the Mn CSV analysis. For the lowest concentrations, average $s$ was 0.0023 , and for the highest concentrations it was 0.011 . Respective low/high prediction intervals were \pm 0.012 (5.3s) and \pm 0.012 (1.1s) for OLS, \pm 0.0049 (2.1s) and \pm 0.023 (2.1s) for eq $13, \pm 0.0054(2.4 s)$ and \pm 0.026 (2.4s) for eq $14, \pm 0.0050$ (2.2s) and \pm 0.019 (1.7s) for eq 15, and $\pm 0.0040(1.7 s)$ and \pm 0.018 (1.6s) for eq 16. However, the middle five concentrations still play a vital role in evaluating the variance functions considering that eq 14 was too conservative at both the low and high end (and therefore too liberal in the middle) while eq 16 was the opposite. While employing any of the VFE equations to these data is acceptable in most practical applications, eqs 13 and 15 are probably best because eq 14 was too sensitive to analyte concentration due its $\mathrm{y}^{2}$ term, whereas eq 16 suffers from the absence of a constant term that is critical at the low end of the calibration. These differences are easier to elucidate in the MCE data than for the PAD and CSV data due to the MCE data covering a full two orders of magnitude in its independent variable despite the MCE variance showing less concentration dependence, as evidenced by the lower $c_{1}$ value in eq 16 . The eq-16 analysis indicates that variance was roughly proportional to the square root of signal, which was a weak dependence relative to many heteroskedastic systems, and therefore may permit the changing variance to be ignored for short calibration ranges (less than a factor of $10 \times$ ).

Monte Carlo simulations of the selenate MCE analysis were performed similarly to those for the other systems. The simulated slope and intercept were 1.13 and 0.000 , respectively. Variance was modeled using eq 16 with $c_{0}=0.00624$ and $c_{1}=0.315$. Calibration concentration ratios were $0.01,0.033,0.1$, 0.33, 1, and 3; test concentration ratios were 0.02 and 2. Regression analyses were performed with OLS and WLS assuming weights of $\mathrm{y}^{-0.63}$ (correct), $\mathrm{y}^{-0.441}$ (30\% low), and $\mathrm{y}^{-0.819}$ (30\% high). Histograms for the OLS and correct WLS prediction intervals are shown in Figure 3e-f. Complete graphical results for the ensemble slope, intercept, best-estimate values, and prediction intervals are included for each 
regression in the supporting information. While qualitatively similar to the simulations for the other two systems, the resulting distributions were noticeably more symmetrical due to the combination of lower concentration dependence for the variance and a smaller concentration range being covered because of the lower standard deviations. Optimal WLS yielded a 9.0\% smaller standard deviation for the slope than OLS, and the $\mathrm{y}^{-0.441}$ and $\mathrm{y}^{-0.819}$ weightings were $8.1 \%$ and $7.7 \%$ smaller, respectively. Similar to the other systems, the measured intercept showed a more substantial improvement, specifically 36.3\%, $34.5 \%$, and $35.1 \%$ for $\mathrm{y}^{-0.63}, \mathrm{y}^{-0.441}$, and $\mathrm{y}^{-0.819}$ weightings, respectively. For the low- and highconcentration points, results were also similar to the other simulations. OLS yielded $0.8 \%$ of the measurements outside of the prediction interval at the low values, but a very high $25.8 \%$ were outside of the interval at the high end. Weightings of $\mathrm{y}^{-0.63}, \mathrm{y}^{-0.441}$, and $\mathrm{y}^{-0.819}$ resulted in low-concentration exception rates of $5.2 \%, 2.9 \%$, and $8.6 \%$ and high-concentration rates of $5.1 \%$, 9.5\%, and $2.4 \%$, respectively. Again, the precision of WLS at the low end of the calibration was substantially better than OLS, with each weighting giving 14-15\% lower standard deviation. Improvements at the high end were less than $1 \%$, again showing that the primary benefit of WLS, other than its improved prediction intervals, occurs at lower concentrations with negligible benefit for high values.

\section{Conclusions}

Variance as a function of analyte signal was characterized through replicate analysis for three emerging miniaturized analytical techniques_-PADs with human-eye detection, electrochemical transparencyfilm analytical devices with CSV detection, and MCE with contact conductivity detection. Even though they cover a broad range of chemistries, instrumental complexity, precision, and detection motifs, each technique exhibited heteroskedastic behavior. The visual PAD devices showed standard deviation to be nearly proportional to concentration (near-constant RSD), the Mn CSV analysis showed standard deviation to be proportional to approximately the square root of concentration, and the MCE system exhibited behavior with the variance being nearly proportional to the square root of concentration. For 
heteroskedastic systems, OLS exhibited suboptimal efficiency and produced prediction intervals that were overestimated at the low end of the calibration and too narrow at the high end. Utilizing WLS produced prediction intervals that were in better agreement with the observed data. The choice of the variance function form had little impact on the shape of the prediction bands for the typical calibration ranges tested.

Monte Carlo simulations of the instrumental responses were performed to quantify the inadequacy of OLS, the advantage of WLS, and the sensitivity of weighted regression to uncertainty in the variance function. Weighted regression was observed to introduce a measurable but practically unimportant bias in the measured slopes and intercepts for two of the techniques. This bias was far outweighed by its improvement in performance-a decrease in the measured standard deviations of the calibration slope by $9-22 \%$ and of the intercept by 33-43\%. Varying the weighting function’s exponent by $30 \%$ showed little drop in performance for measuring the slope and intercept. Representative "sample” values were also evaluated in the simulations. OLS was again outperformed by weighted regression in terms of precision, with the lower concentrations benefiting considerably more than higher concentrations. Proper weightings produced close to the expected 5\% of the measurements falling outside of the $95 \%$ prediction intervals at both ends of the calibration, whereas ordinary least squares showed $0.5-0.8 \%$ of the low-concentration points outside of the intervals and $13-26 \%$ of the high points. Improper weightings still gave better results than unweighted regression, with the number of points outside the prediction intervals within about a factor of two of the expected values.

Overall, this work is the first characterization of the variance functions for PADs and MCE systems. Weighted least-squares regression should be employed for each of the tested techniques to improve instrumental precision and the generated uncertainty estimates. Because the choice of the variance function has only a small effect on the results for typical calibration ranges and uncertainty in the variance function coefficients still yields performance superior to OLS, a power model of variance (eq 16) may be the best variance-function choice, as it is effectively a one-parameter model that is easily 
employed. However, this model may perform poorly near the limit of detection, and ultimately the model chosen in practice may be application dependent. 


\section{Acknowledgement}

Parts of this work were funded by U.S. National Science Foundation (NSF) grant 1415655 under contract from Access Sensor Technologies. 


\section{References}

[1] Tellinghuisen, J., Least Squares with Non-Normal Data: Estimating Experimental Variance Functions, Analyst 133 (2008) 161-166.

[2] Thompson, M., Variation of Precision with Concentration in an Analytical System, Analyst 113 (1988) 1579-1587.

[3] Tellinghuisen, J., Weighted Least-Squares in Calibration: What Difference Does It Make?, Analyst 132 (2007) 536-543.

[4] Davidian, M., Carroll, R. J., Variance Function Estimation, J. Am. Stat. Assoc. 82 (1987) 10791091.

[5] Davidian, M., Haaland, P. D., Regression and Calibration with Nonconstant Error Variance, Chemometr. Intell. Lab. 9 (1990) 231-248.

[6] Tellinghuisen, J., Variance Function Estimation by Replicate Analysis and Generalized Least Squares: A Monte Carlo Comparison, Chemometr. Intell. Lab. 99 (2009) 138-149.

[7] Zorn, M. E., Gibbons, R. D., Sonzogni, W. C., Weighted Least-Squares Approach to Calculating Limits of Detection and Quantification by Modeling Variability as a Function of Concentration, Anal. Chem. 69 (1997) 3069-3075.

[8] Thompson, M., Coles, B., Examples of the 'Characteristic' Function Applied to Instrumental Precision in Chemical Measurement, Accred. Qual. Assur. 14 (2009) 147-150.

[9] Cate, D. M., Dungchai, W., Cunningham, J. C., Volckens, J., Henry, C. S., Simple, DistanceBased Measurement for Paper Analytical Devices, Lab Chip 13 (2013) 2397-2404.

[10] Cate, D. M., Noblitt, S. D., Volckens, J., Henry, C. S., Multiplexed Paper Analytical Device for Quantification of Metals Using Distance-Based Detection, Lab Chip 15 (2015) 2808-2818.

[11] Berg, K. E., Adkins, J. A., Boyle, S. E., Henry, C. S., Manganese Detection Using StencilPrinted Carbon Ink Electrodes on Transparency Film, Electroanalysis (2015) submitted.

[12] Noblitt, S. D., Schwandner, F. M., Hering, S. V., Collett, J. L., Henry, C. S., High-Sensitivity Microchip Electrophoresis Determination of Inorganic Anions and Oxalate in Atmospheric Aerosols with Adjustable Selectivity and Conductivity Detection, J. Chromatogr. A 1216 (2009) 1503-1510.

[13] Noblitt, S. D., Staicu, L. C., Ackerson, C. J., Henry, C. S., Sensitive, Selective Analysis of Selenium Oxoanions Using Microchip Electrophoresis with Contact Conductivity Detection, Anal. Chem. 86 (2014) 8425-8432.

[14] Brunetti, B., Cattaneo, R., Desimoni, E., Electroanalytical Quantification of Rhodium in Lentils Exposed to Increasing Rh(Iii) Concentrations, Italian J. Food Sci. 17 (2005) 437-446.

[15] Desimoni, E., Brunetti, B., About Estimating the Limit of Detection of Heteroscedastic Analytical Systems, Anal. Chim. Acta 655 (2009) 30-37.

[16] Baumann, K., Wätzig, H., Appropriate Calibration Functions for Capillary Electrophoresis Ii. Heteroscedasticity and Its Consequences, J. Chromatogr. A 700 (1995) 9-20.

[17] del Río Bocio, F. J., Riu, J., Boqué, R., Rius, F. X., Limits of Detection in Linear Regression with Errors in the Concentration, J. Chemometr. 17 (2003) 413-421.

[18] Alnajjar, A. O., Validation of a Capillary Electrophoresis Method for the Simultaneous Determination of Amlodipine Besylate and Valsartan in Pharmaceuticals and Human Plasma, J. AOAC Int. 94 (2011) 498-502.

[19] Horstkötter, C., Kober, S., Spahn-Langguth, H., Mutschler, E., Blaschke, G., Determination of Triamterene and Its Main Metabolite Hydroxytriamterene Sulfate in Human Urine by Capillary Electrophoresis Using Ultraviolet Absorbance and Laser-Induced Fluorescence Detection, J. Chromatogr. B 769 (2002) 107-117.

[20] Santoyo, E., García, R., Aparicio, A., Verma, S. P., Verma, M. P., Evaluation of Capillary Electrophoresis for Determining the Concentration of Dissolved Silica in Geothermal Brines, J. Chromatogr. A 1071 (2005) 197-204.

[21] Toasaksiri, S., Massart, D. L., Heyden, Y. V., Study of Method Validation Criteria in a Capillary Electrophoresis Method for the Separation of Non-Steroidal Anti-Inflammatory Drugs, Anal. Chim. Acta 416 (2000) 29-42. 
[22] Miller, J. N., Basic Statistical-Methods for Analytical-Chemistry .2. Calibration and Regression Methods - a Review, Analyst 116 (1991) 3-14.

[23] Garden, J. S., Mitchell, D. G., Mills, W. N., Non-Constant Variance Regression Techniques for Calibration-Curve-Based Analysis, Anal. Chem. 52 (1980) 2310-2315.

[24] Almeida, A. M., Castel-Branco, M. M., Falcão, A. C., Linear Regression for Calibration Lines Revisited: Weighting Schemes for Bioanalytical Methods, J. Chromatogr. B 774 (2002) 215-222.

[25] Horwitz, W., Kamps, L. R., Boyer, K. W., Quality Assurance in the Analysis of Foods for Trace Constituents, J. Assoc. Off. Ana. Chem. 63 (1980) 1344-1354.

[26] Duffy, D. C., McDonald, J. C., Schueller, O. J. A., Whitesides, G. M., Rapid Prototyping of Microfluidic Systems in Poly(Dimethylsiloxane), Anal. Chem. 70 (1998) 4974-4984.

[27] Noblitt, S. D., Henry, C. S., Improving the Compatibility of Contact Conductivity Detection with Microchip Electrophoresis Using a Bubble Cell, Anal. Chem. 80 (2008) 7624-7630.

[28] Noblitt, S. D., Henry, C. S., Calibration-Free Quantitation in Microchip Zone Electrophoresis with Conductivity Detection, Electrophoresis 36 (2015) 1927-1934.

[29] Jacobson, S. C., Koutny, L. B., Hergenroder, R., Moore, A. W., Ramsey, J. M., Microchip Capillary Electrophoresis with an Integrated Postcolumn Reactor, Anal. Chem. 66 (1994) 3472-3476.

[30] Altria, K. D., Improved Performance in Capillary Electrophoresis Using Internal Standards, LC GC Europe 15 (2002) 588-594.

[31] Mayer, B. X., How to Increase Precision in Capillary Electrophoresis, J. Chromatogr. A 907 (2001) 21-37.

[32] Bidulock, A. C. E., van den Berg, A., Eijkel, J. C. T., Improving Chip-to-Chip Precision in Disposable Microchip Capillary Electrophoresis Devices with Internal Standards, Electrophoresis 36 (2015) 875-883.

[33] Dose, E. V., Guiochon, G. A., Internal Standardization Technique for Capillary Zone Electrophoresis, Anal. Chem. 63 (1991) 1154-1158. 
Abstract Figure

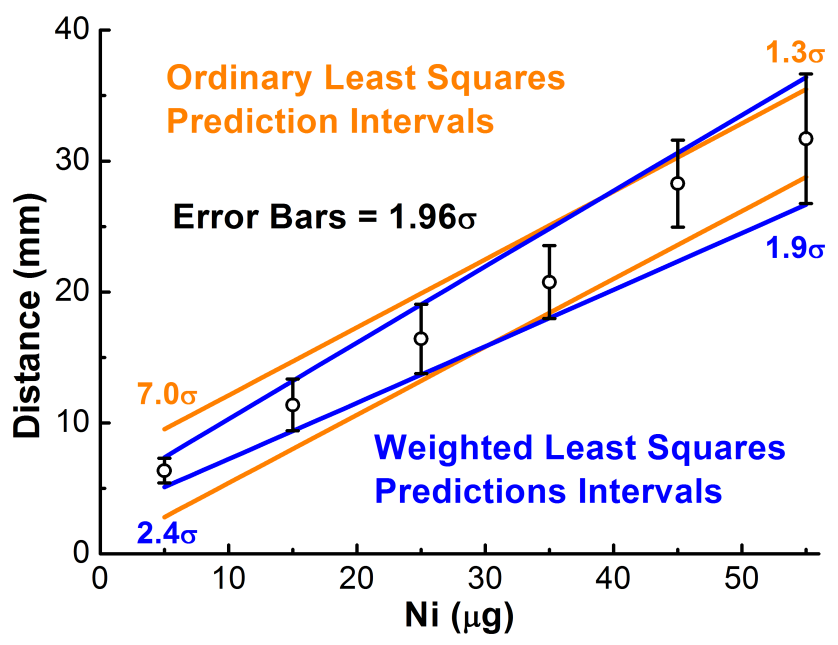

